\title{
The effect of mass transfer on steady two-dimensional rimming flow
}

\author{
Caroline J. Noakes · John R. King • \\ David S. Riley
}

Received: 10 February 2010 / Accepted: 2 November 2010 / Published online: 17 November 2010

(C) The Author(s) 2010. This article is published with open access at Springerlink.com

\begin{abstract}
Motivated by applications in rapidly rotating machinery, the standard lubrication model of twodimensional thin-film flow on the inside of a rotating circular cylinder is adapted in a simple manner to incorporate the effect of mass flux into the free surface of the film. A numerical attack, validated against published results for the zero-mass-flux case, is used to study the case of constant mass fraction with non-zero injection into and extraction from the film, this representing the steady-state operating condition in an aero-engine. It is found that increasing mass flux inhibits the formation of the steep fronts on the film surface, thus reducing the chances of droplets being stripped away from the film when there is a core flow present, and that recirculation zones may be suppressed by an increase in the Bond number, thus reducing the chances of oil degradation in rapidly rotating machinery.
\end{abstract}

Keywords Lubrication analysis · Mass sources $\cdot$ Numerical computations $\cdot$ Rimming flow

\section{Introduction}

It is well known that a complex variety of fluid-dynamical behaviour arises when a horizontal circular cylinder partially filled with a simple Newtonian fluid is rotated; see for example [1]. There is an ever-growing literature on the mathematical analysis of this so-called rimming-flow problem: in particular, [2-5] focus on a simple lubrication model; [6-11] consider various higher-order theories; some in addition investigate surface-tension effects. More recent studies, [12-14] have focussed on inertial effects, while others, [15-17], have used intricate multipletimescale analysis to describe the evolution of instabilities in rimming flow. (Some of the papers cited here in turn cite many more papers, many of which are concerned with coating flow, the flow on the exterior of a cylinder. For our purposes this brief list of references is sufficient.)

In the simplest model of rimming flow, a balance between viscosity and gravity is assumed and the effects of inertia and surface tension are neglected. Applying the ideas of lubrication theory to this situation, Moffatt [2] studied the maximum load of liquid that can be supported on the outside of a cylinder (this particular problem strictly belongs to the complementary class of coating flows, but his analysis applies in both situations with appropriate modifications in interpretation). The problem of rimming flow was revisited in [4] and [7], with both addressing the question of the existence of weak solutions containing shocks (where the free-surface height jumps between a

C. J. Noakes · J. R. King · D. S. Riley $(\varangle)$

Division of Applied Mathematics, School of Mathematical Sciences, University of Nottingham, Nottingham NG7 2RD, UK

e-mail: david.riley@nottingham.ac.uk 
relatively low value and a relatively large value in a very narrow region of the domain). It was Johnson [4] who first recognized the relevance of discontinuous solutions of the equation determining the steady-state film profile as a function of the azimuthal angle. Benjamin et al. [7] made a deeper study and, inter alia, considered the stability of the solutions that [4] had identified and the influence of surface tension, the latter effect being investigated in more detail in [6]. A more recent, comprehensive study of the effects of surface tension and higher-order gravitational effects has been undertaken by Villegas-Díaz et al. [18] in a study of rimming flow subject to constant surface shear with the aim of resolving issues regarding existence, uniqueness and stability of such weak solutions.

It is the behaviour of an oil film that forms on the inside of the outer shaft of an aero-engine bearing chamber that is the primary concern in this work. The fluid in the body of the chamber is a mixture of air and oil, driven round by the rotation of a central shaft. Oil enters the chamber from an injector, forms a mist in the air and eventually impacts on the outer wall as small droplets. The oil's function is to act as a lubricant for the bearings, and also as a coolant of the hot metal surfaces. Thus it is important that a constant flow of oil is maintained around the wall, since stagnant or dry patches may lead to oil degradation and, in very extreme cases, to coking. Oil leaves the chamber through locations on the outer cylindrical wall and collects in external sumps.

Thus the problem involves the study of the flow of a thin film of oil on the inside of a smooth rigid rotating cylinder under the influences of gravity and a core flow consisting of a rotating mixture of air and oil droplets. Oil leaves the chamber through a sink on the cylinder wall. In reality the droplets interact with the film in a complex manner, rebounding, splashing and breaking up, as well as being absorbed; see [19-21]. As a starting point in developing our understanding, it is sensible to study a very simple continuous droplet distribution, obtained by averaging the droplet and air-core flows spatially, rather than a discrete one. Moreover, given the complexity of the full problem, it is reasonable to focus on the effect of mass flux in isolation, and initially ignore momentum transfer.

In this scoping study, the effects of such mass transfer on the steady two-dimensional rimming flows described by previous workers using lubrication theory are investigated. A simple model of injection into and draining from the film is developed and solved numerically. We consider the special case in which gravity, viscous and surface-tension effects, though weak, are in balance.

The plan of the paper is as follows. The simple model incorporating fluid inflow to and outflow from the film is constructed in Sect. 2 and solved numerically. This is followed, in Sect. 3, by a summary of known results for steady flows without surface tension, found first by Moffatt [2], and a comparison with published results on the smoothing effects of surface tension, [22]; streamlines are discussed in Sect. 4. The main part of the study is reported in Sect. 5 where the flows are subjected to injection and extraction of fluid. Finally, in Sect. 6 we discuss our conclusions.

\section{Mathematical model, numerical scheme and solution method}

The mathematical basis is taken to be the thin, small reduced-Reynolds-number film model discussed first by Pukhnachev [3] and, in the absence of surface tension, by Moffatt [2]. Although rimming flows are considered here, the governing profile equations also hold for coating flows (i.e., flows on the exterior surface of the cylinder). This study adopts a pragmatic approach to an extremely complex and challenging practical problem and the description of the model development follows the spirit of [7], rather than the formal asymptotic approach of [6] or [18], for example.

The geometry of the problem to be considered is illustrated in Fig. 1, where the physical parameters (all assumed constant) are the radius of the cylinder, $a$, the viscosity of the fluid, $\mu$, the density of the fluid, $\rho$, the angular speed of rotation, $\Omega$, and the acceleration due to gravity, $g$; $\tilde{h}$ denotes the thickness of the fluid layer. Standard polar coordinates $r$ and $\theta(0 \leq \theta<2 \pi)$, where the angular position is measured in the direction of rotation from the downward vertical, are used (note that some of the authors cited above measure the angular position from the equator on the rising side of the cylinder) and $\tilde{t}$ denotes time (although the current focus is on steady states, a time-dependent approach is adopted to (i) test implicitly for stability, (ii) allow for different initial film profiles, and (iii) be easily extendable to time-dependent filling problems; see [9]). The free surface of the fluid is thus located at $r=a-\tilde{h}$. 
Fig. 1 Schematic of a rimming flow on the inner surface of a horizontal rotating cylinder

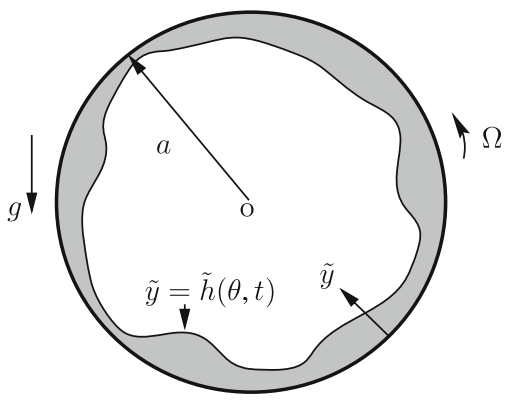

According to lubrication theory $\left(\delta \equiv(\nu \Omega / a g)^{\frac{1}{2}} \rightarrow 0\right)$, only the azimuthal velocity component $\tilde{v}$ is significant dynamically. On neglecting inertial effects (reduced Reynolds number is small), the dynamic pressure $\tilde{p}$ in the fluid is constant across the film, being given by the normal-stress condition at the free surface:

$\tilde{p}=-\frac{T}{a^{2}}\left(\tilde{h}+\frac{\partial^{2} \tilde{h}}{\partial \theta^{2}}\right)$,

where $T$ denotes the surface-tension coefficient. The key dynamical equation is

$\mu \frac{\partial^{2} \tilde{v}}{\partial \tilde{y}^{2}}=\rho g \sin \theta+\frac{1}{a} \frac{\partial \tilde{p}}{\partial \theta}$.

Here $\mu$ denotes the coefficient of viscosity, $\tilde{y}=a-r$ is the distance from the cylinder wall and, at this boundary wall, the no-slip condition requires

$\tilde{v}=\Omega a$ at $\tilde{y}=0$,

while at the interface,

$\frac{\partial \tilde{v}}{\partial y}=0$ at $\tilde{y}=\tilde{h}$

It thus follows that

$\tilde{v}=\Omega a-\left(\frac{g \sin \theta}{v}+\frac{1}{a \mu} \frac{\partial \tilde{p}}{\partial \theta}\right)\left(\tilde{h} \tilde{y}-\frac{1}{2} \tilde{y}^{2}\right)$,

where $v$ denotes the fluid's kinematic viscosity.

As previously mentioned, methods of transferring fluid to the film include droplets or a jet of fluid impacting at the free surface and, from the film, scavenges and film breakup. To model these phenomena accurately would require a highly complex model accounting for the coalescence of the droplets or of the jet with the film as well as effects such as splashing. This is beyond the scope of the current investigation and, since only the general effect of fluid inflow/outflow on the film profile is of interest here, a much simpler model is adopted. The injection/extraction of fluid is included through incorporating flux functions into the formulation of mass balance. We thus adopt the idealised volume-conservation law

$\frac{\partial \tilde{h}}{\partial \tilde{t}}+\frac{1}{a} \frac{\partial \tilde{q}}{\partial \theta}=\tilde{\lambda}_{I} I_{I}(\theta)-\tilde{\lambda}_{O} I_{O}(\theta)$

where $\tilde{q}$, the azimuthal flux of fluid per unit axial depth, is given by

$\tilde{q}=\Omega a \tilde{h}-\frac{1}{3} \tilde{h}^{3}\left(\frac{g \sin \theta}{v}+\frac{1}{a \mu} \frac{\partial \tilde{p}}{\partial \theta}\right)$,

and $\tilde{I}_{I, O}(\theta)$ are dimensionless functions describing the steady distribution of inflow and outflow around the cylinder, the subscripts $I$ and $O$ denoting inflow and outflow, respectively. The parameters $\tilde{\lambda}_{I}$ and $\tilde{\lambda}_{O}$ represent the flow rates. 
The natural scales for non-dimensionalising the problem follow from examining (5)-(7). On introducing

$\tilde{y}=(\nu \Omega a / g)^{\frac{1}{2}} y, \quad \tilde{h}=(\nu \Omega a / g)^{\frac{1}{2}} h, \quad \tilde{p}=\rho g a p, \quad \tilde{v}=\Omega a v$,

$\tilde{t}=\Omega^{-1} t, \quad \tilde{q}=\left(v \Omega^{3} a^{3} / g\right)^{\frac{1}{2}} q, \quad \tilde{\lambda}_{I}=\left(v \Omega^{3} a^{3} / g\right)^{\frac{1}{2}} \lambda_{I}, \quad \tilde{\lambda}_{O}=\left(v \Omega^{3} a^{3} / g\right)^{\frac{1}{2}} \lambda_{O}$,

the key equations become

$$
\begin{aligned}
& v(\theta, t)=1-\left(h y-\frac{1}{2} y^{2}\right)\left(\sin \theta-\epsilon\left(h+\frac{\partial^{2} h}{\partial \theta^{2}}\right)_{\theta}\right), \\
& q(\theta, t)=h-\frac{1}{3} h^{3}\left(\sin \theta-\epsilon\left(h+\frac{\partial^{2} h}{\partial \theta^{2}}\right)_{\theta}\right), \\
& \frac{\partial h}{\partial t}+\frac{\partial q}{\partial \theta}=\lambda I(\theta),
\end{aligned}
$$

where $\lambda I(\theta)=\lambda_{I} I_{I}(\theta)-\lambda_{O} I_{O}(\theta)$ represents the total local inflow into the film and $\epsilon$ is the Bond number defined by $\epsilon=\frac{T}{\rho g a^{3}}\left(\frac{\nu \Omega a}{g}\right)^{\frac{1}{2}}$.

Different representations of $I$ will be considered in the following sections; in general terms, where $I$ is positive fluid flows into the film and where $I$ is negative fluid flows out of the film.

Equation (11) was solved numerically using the method of lines, [23], with the discretised system being given by

$$
\begin{aligned}
& \frac{\partial h_{i}}{\partial t}=-\left(\frac{q_{i}-q_{i-1}}{\Delta \theta}\right)+\lambda I_{i}, \\
& q_{i}=\epsilon \frac{h_{i}^{3}}{3}\left(\frac{h_{i+2}-2 h_{i+1}+2 h_{i-1}-h_{i-2}}{2 \Delta \theta^{3}}+\frac{h_{i+1}-h_{i-1}}{2 \Delta \theta}\right)-\frac{h_{i}^{3}}{3} \sin \theta_{i}+h_{i},
\end{aligned}
$$

where central differences have been used for derivatives in (10), while a backward-difference scheme is used for the derivative of $q$ in (11). Due to the periodic nature of the grid, we have

$q_{0}=q_{n-1}, \quad h_{0}=h_{n-1}, \quad h_{-1}=h_{n-2}, \quad h_{n+1}=h_{2}, \quad h_{n}=h_{1}, \quad I_{n}=I_{1}$,

which are needed when evaluating $q_{1}, q_{2}$ and $q_{n-1} . n$ is the number of grid points, with $(n-1) \Delta \theta=2 \pi$, and $\theta_{i}=(i-1) \Delta \theta$. The system has been solved numerically using the NAG routine D02EJF, which integrates a stiff set of first-order differential equations using a variable-order, variable-step method.

\section{Zero mass injection and extraction}

The steady-state solutions of the system with zero surface tension are well known and were originally described in [2] in terms of the value of the flux $q$ and a dimensionless mean film thickness $\bar{h}$ defined by

$\bar{h}=\frac{1}{2 \pi} \int_{0}^{2 \pi} h(\theta, t) \mathrm{d} \theta$,

which is effectively a measure of the volume fraction. When $q<2 / 3$, there is a single, bounded, neutrally stable film profile which is smooth and completely wets the cylinder's surface. This profile has vertical symmetry, its thickness having a maximum at $\theta=\pi / 2$, i.e., at the equator on the rising side of the cylinder, and a minimum at the point diagonally opposite. When $q=2 / 3$ and $\bar{h}=\bar{h}_{c 1}=0.7071$, the profile remains symmetric and continuous, but loses smoothness, having a discontinuous slope at $\theta=\pi / 2$. At this point a second unphysical, unbounded branch effectively coalesces with the physical branch. For $q=2 / 3$ and $\bar{h}_{c 1}<\bar{h}<\bar{h}_{c 2}$, where $\bar{h}_{c 2}=1.1023$, no continuous profile exists, but asymmetric stable (weak) solutions can be constructed from the two solution branches by introducing a shock in the first quadrant (i.e., by jumping between the two branches). (The research literature 

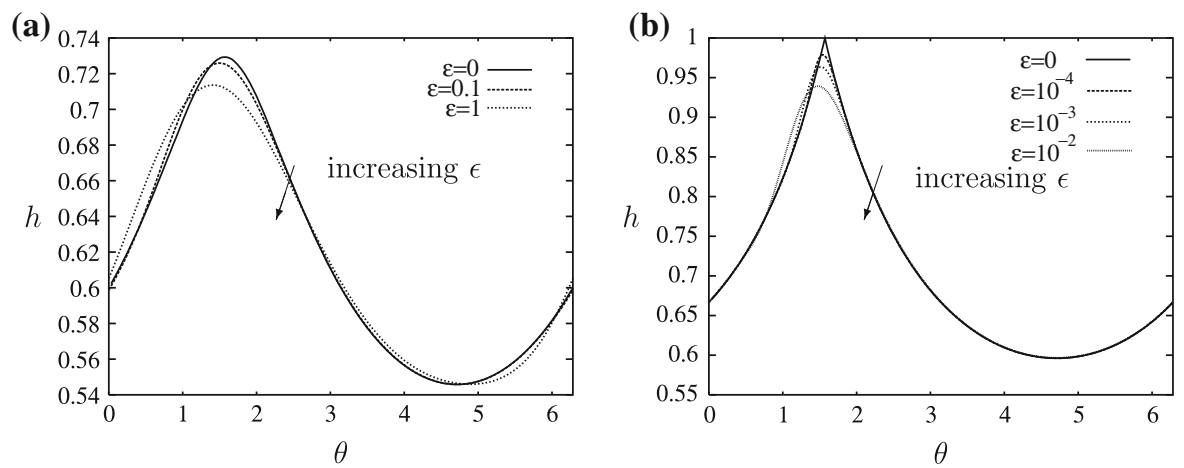

Fig. 2 Steady-state profiles for $\mathbf{a} \bar{h}=0.6169<\bar{h}_{c 1}$, b $\bar{h}=\bar{h}_{c 1}=0.7071$. The profiles for non-zero values of $\epsilon$ were obtained numerically, while the $\epsilon=0$ profile is the algebraic solution to (10)

contains several attempts to describe, theoretically and numerically, the regions around such shocks, whose existence is formally incompatible with the underlying lubrication assumptions. Interesting questions remain from the point of view of asymptotic analysis of the shock regions; see [18] and [24]. Notwithstanding the lack of a complete asymptotic description, numerical evidence suggests that the lubrication description is surprisingly accurate.) Benjamin et al. [7] were the first to highlight and calculate the upper bound corresponding to $\bar{h}_{c 2}$; note that their calculated value of 1.1067, which is in agreement with that stated in [10], is slightly inaccurate; see [11].

We first investigate numerically the effect of weak surface tension on these known solutions. The initial profile was taken to be uniform and the subsequent profiles were output at regular time intervals. The profile was assumed to have reached a steady-state when the height of the film at each grid point at consecutive time steps was unchanged within a tolerance of $10^{-7}$. Mass conservation was checked at each output stage by summing the values of $h$ at each grid point with the result being constant to eight decimal places throughout the computation. The program was also checked by solving the case of zero gravity, when the solution to the governing equations is a steady, uniform film. This was found to result for large time when the sine term in (13) was replaced by zero, regardless of the thickness of the initial film profile. After successive runs with various numbers of mesh points, it was found that 1,000 were needed to ensure a numerically stable solution, while keeping computation time at a reasonable level.

Figure 2a shows results for $\bar{h}=0.6169<\bar{h}_{c 1}$, for various values of $\epsilon$, along with the corresponding analytical result for zero surface tension. For small $\epsilon\left(<10^{-2}\right)$ the inclusion of surface tension has no noticeable effect on the film profile. However, as $\epsilon$ is increased (stronger surface-tension forces), the extent of the increase in the film thickness at the mid-point of the rising side of the cylinder is reduced. Also, the points of maximum and minimum thickness are displaced in the direction of $\theta=0$ (the bottom of the cylinder), thus breaking the symmetry of the profile about $\theta=\pi / 2$.

When $\bar{h}=\bar{h}_{c 1}$ the effects of surface tension are more evident for larger values of $\epsilon$, as shown in Fig. 2b. The corner profile that results in the case of zero surface tension is smoothed and again the maximum height is reduced and displaced towards the bottom of the cylinder as $\epsilon$ is increased.

When $\bar{h}$ is in excess of $\bar{h}_{c 1}$, a steep front is seen to develop, as illustrated in Fig. 3 for $\bar{h}=0.7293$ and various values of $\epsilon$. Surface tension smooths out the discontinuity, displacing the peak towards $\theta=\pi / 2$ and decreasing its height. However, for very small values of $\epsilon\left(\sim 10^{-5}\right)$ the height of the front is increased slightly, although the curvature at the top of the front is still reduced due to the surface tension. Additionally, for small values of $\epsilon$, a capillary wave is evident at the base of the front. The increase in height of the front for small $\epsilon$ is required to compensate for the reduction in volume of fluid at the base of the front due to the capillary wave. This description accords well with the results of the study by Benilov et al. [25], for example see their Fig. 5b. Moreover these results are wholly consistent with those of [26] and also with those of [7], although their model included the terms of $O(\delta)$ neglected here and surface-tension terms were assumed also to be of that order. 
Fig. 3 Steady-state profiles for $\bar{h}_{c 1}<\bar{h}=0.7293<\bar{h}_{c 2}$. The profiles for non-zero values of $\epsilon$ were obtained numerically, while the $\epsilon=0$ profile is the solution to (10)

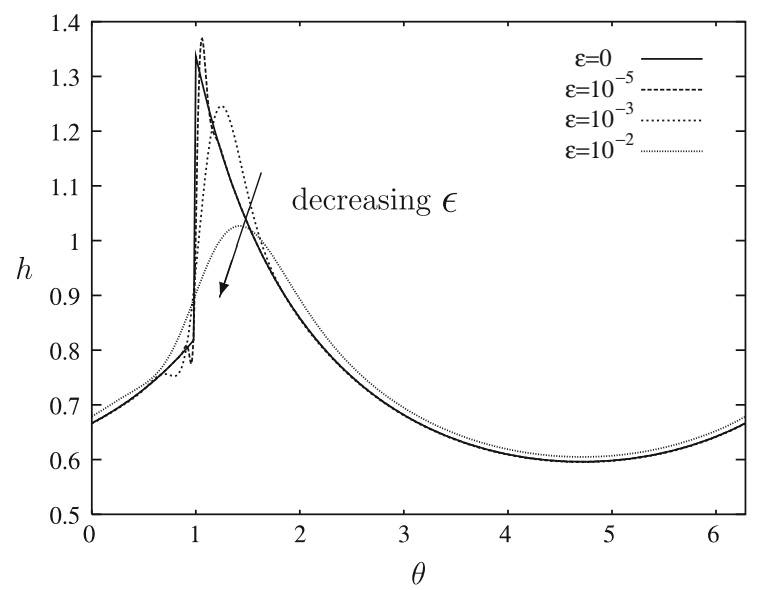

(a)

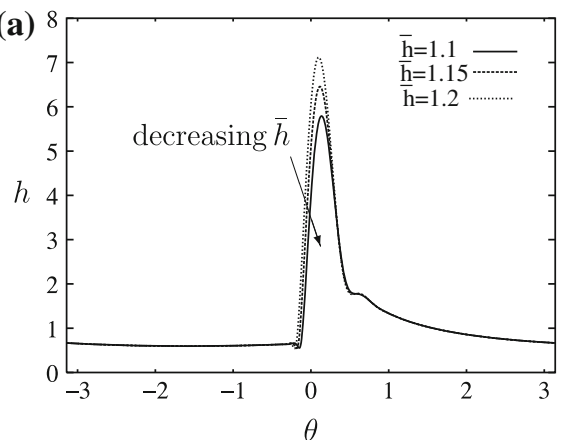

(b)

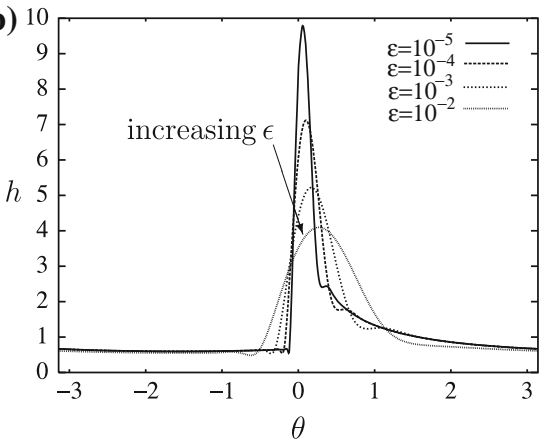

Fig. 4 Steady-state profiles obtained numerically for $\mathbf{a} \epsilon=10^{-4}$ and $\bar{h}=1.1,1.15$ and $1.2, \mathbf{b} \bar{h}=1.2>\bar{h}_{c 2}$ and $\epsilon=10^{-5}, 10^{-4}, 10^{-3}$ and $10^{-2}$

While Moffatt [2] showed that the zero-surface-tension case exhibits an upper limit $\bar{h}_{c 2}$ for $\bar{h}$, the numerical results for non-zero surface tension suggest no such bound, which is consistent with the theoretical result of [24] for the special case of constant shear at the film surface. When $\bar{h}$ is increased beyond $\bar{h}_{c 2}$, with surface tension non-zero, the front remains at $\theta=0$, as shown in Fig. 4a. The height of the peak increases to accommodate the extra volume, while the rest of the profile remains essentially unchanged from that of $\bar{h}=\bar{h}_{c 2}$, except for capillary waves at the base of the peak; cf. [22, Fig. 11] in which $\bar{h}=\bar{h} \approx 1.512$ in the present notation. Again, increasing surface tension has the effect of smoothing out the profile; see Fig. 4b. It should be noted that the graphical representation of the film profile exaggerates the height of the peak. In fact, in the configuration of the cylinder, these large volume flows represent a pool of liquid at the bottom of the cylinder with a thin film over the remainder of the surface. These solutions are investigated analytically in [6,9], and [22].

Similar profiles for large volumes have been obtained by Tirumkudulu and Acrivos [10], although surface-tension effects were not included in their analysis. Instead, arguing from a physical standpoint, they introduced into the model of [7] an asymptotically non-systematic pressure modification term due to gravity to allow for the evolution of the profile for $\bar{h}>\bar{h}_{c 2}$. Their results feature the development of a pool at the bottom of the cylinder with a thin film around the remainder of the surface, which they show to agree very favourably with experimental results and also with solutions to the full Stokes equations.

\section{Streamlines of the flow}

The streamlines of the flow may be investigated via the introduction of a stream function $\psi(y, \theta)$ given by 

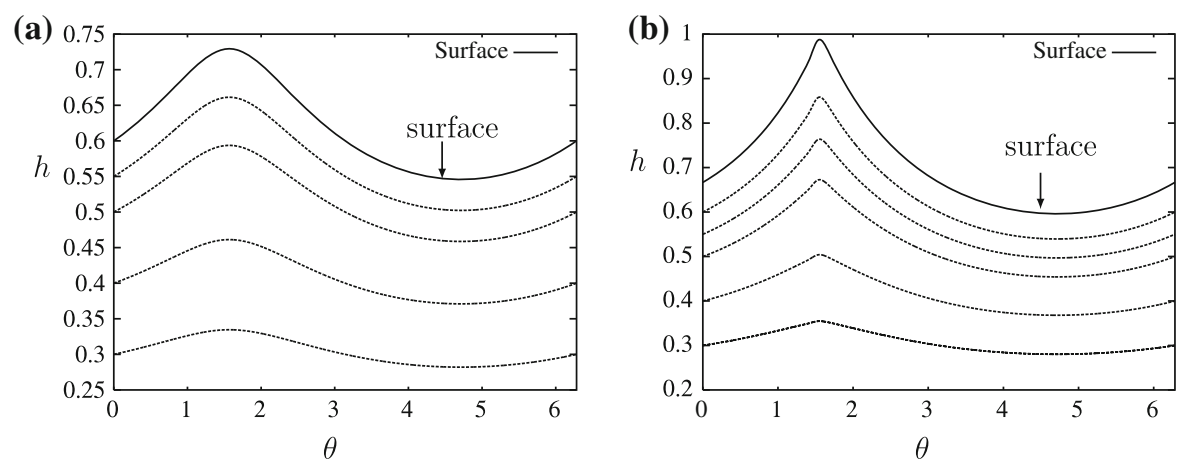

Fig. 5 Streamlines for $\mathbf{a} \bar{h}=0.6169, \epsilon=10^{-3}, \mathbf{b} \bar{h}=\bar{h}_{c 1}, \epsilon=10^{-5}$
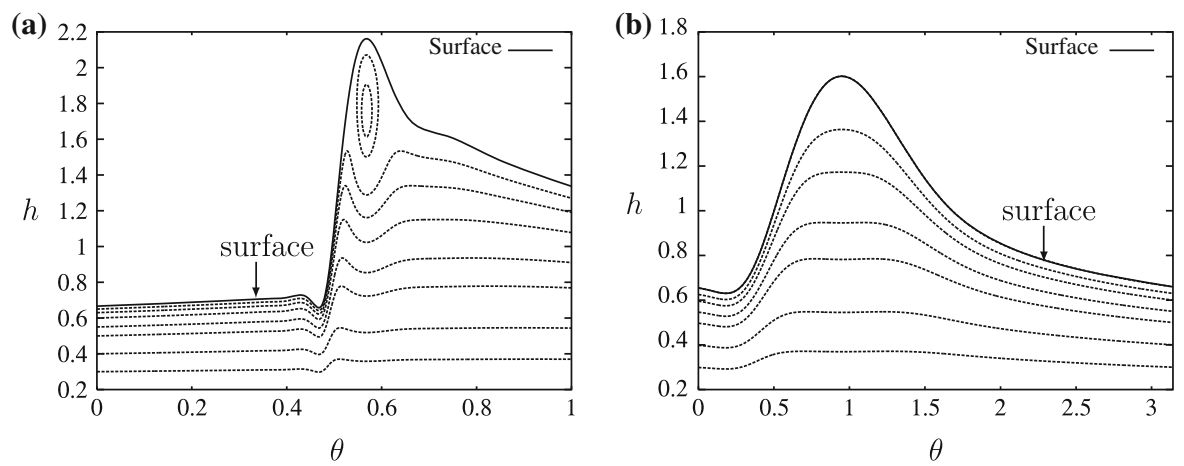

Fig. 6 Streamlines for $\mathbf{a} \bar{h}=0.7978, \epsilon=10^{-5}(q=0.667), \mathbf{b} \bar{h}=0.7978, \epsilon=10^{-2}(q=0.745)$. The recirculation zone is suppressed by the increase in $\epsilon$; the full range of $\theta$ is not shown

$\psi(y, \theta)=\frac{3(q-h)}{h^{3}}\left(\frac{y^{2} h}{2}-\frac{y^{3}}{6}\right)+y$.

The streamlines for various steady-state flows are plotted using the numerical results from the previous section. For $\bar{h} \leq \bar{h}_{c 1}$ (Fig. 5) the streamlines essentially follow the surface of the film, regardless of the value of $\epsilon$. When $\bar{h}_{c 1}<\bar{h}<\bar{h}_{c 2}$, the steep front profile is evident in Fig. 6a, and, as noted by Johnson [4] for the case of zero surface tension, a region of recirculating fluid may develop within the front; compare also [22, Fig. 4] in which again $\bar{h} \approx 1.512$ in the present notation. In terms of bearing-chamber film flows, regions of recirculating fluid are undesirable since the oil is also required to act as a cooling agent within the chamber and a pool of recirculating fluid will inhibit the dissipation of heat. Johnson [4] showed that the occurrence of this recirculation region is dependent on the value of $h_{\max }$, the maximum height of the film, which in turn is dependent on the value of $\bar{h}$. It was illustrated above that, with the introduction of surface tension, $h_{\max }$ is also dependent on the Bond number $\epsilon$ and, as evidenced by Fig. 6b, the recirculating zone may be suppressed by increasing $\epsilon$.

For a zone of recirculation to be present there must exist a value of $y$ in the interval $\left[0, h_{\max }\right)$ where

$\frac{\partial \psi}{\partial y}=0$

i.e., in this case

$y=h_{\max } \pm \sqrt{h_{\max }^{2}+\frac{2 h_{\max }^{3}}{3\left(q-h_{\max }\right)}}$. 

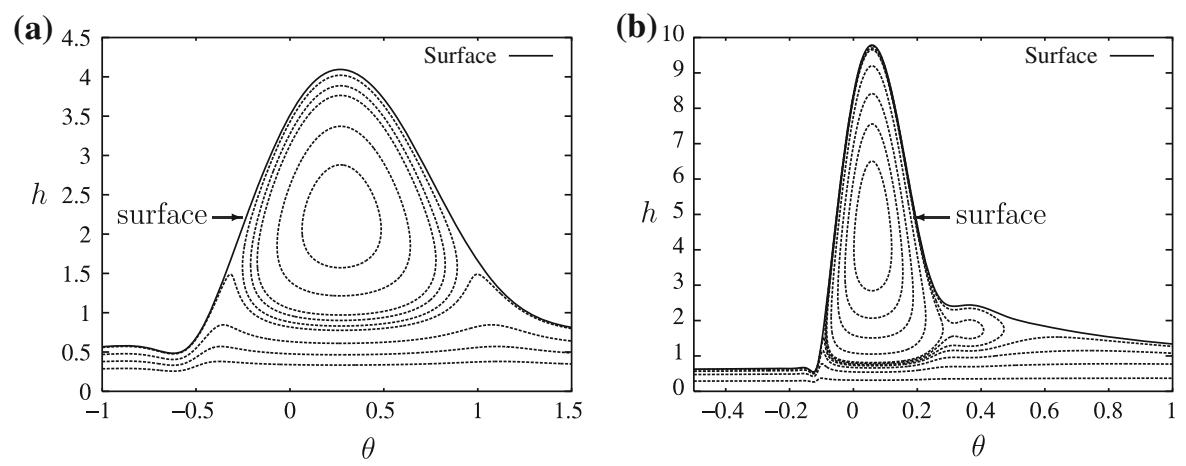

Fig. 7 Streamlines for $\mathbf{a} \bar{h}=1.2, \epsilon=10^{-2}, \mathbf{b} \bar{h}=1.2, \epsilon=10^{-5}$; the full range of $\theta$ is not shown

The value of $q$ may be determined from the numerical results; of course, as $\epsilon \rightarrow 0, q \rightarrow 2 / 3$. For valid solutions $y$ must be less than $h_{\max }$ (implying the negative square root) and

$h_{\max }^{2}+\frac{2 h_{\max }^{3}}{3\left(q-h_{\max }\right)}>0$,

i.e., $h_{\max }>3 q$ for a zone of recirculation to be present. After appropriate rescaling, this result is equivalent to the condition determined by Johnson [4] for recirculation when the surface tension is zero. The numerical results are consistent with this condition, as illustrated in Fig. 6 for $q=0.667$ and 0.745 , respectively.

For $\bar{h}>\bar{h}_{c 2}$, the recirculating zone is more evident, with a large pool present at the bottom of the cylinder beneath which the remainder of the fluid flows; see Fig. 7. For small $\epsilon$ there also appears to be a secondary region of recirculation to the right of the main pool, as illustrated in Fig. 7b.

\section{Constant volume flows—combined injection and extraction}

Various scenarios can be modelled by choosing different expressions for the input function $I$ and different values of the flow-rate parameter $\lambda$. For example, Noakes [9] investigated the unsteady filling process when $I_{I}>I_{O}$ and the mass fraction increases. In the following sections the development of the film profile is investigated when fluid is continuously added through the film surface and extracted through the cylinder wall such that the volume of fluid in the film remains fixed. Both uniform and non-uniform distributions of fluid inflow, representing the mass transfer that may occur due to impacting droplets or a jet of fluid, are considered.

In all the cases which follow it can be readily shown numerically that, if the mass transfer is switched off, the film profile returns to the steady-state profile corresponding to the volume of fluid remaining in the flow.

Fluid in the film is extracted by draining it out through the cylinder wall. For simplicity, extraction of film fluid is modelled through a single region of outflow located over $1 \%$ of the cylinder's surface at $\theta=0$ (the bottom of the cylinder). This corresponds to an idealisation of a scavenge that drains off fluid in the lower half of an aero-engine bearing chamber. With the incorporation of such a region of fluid extraction, the volume of fluid within the film may be kept constant and steady-state flows may be achieved.

Two models of injection are now considered.

\subsection{Uniform injection with a fixed point of extraction}

Within an aero-engine bearing chamber a large number of fluid droplets may be present in the rotating core air flow. Some of these droplets will inevitably find their way into the films flowing on the surfaces of the chamber, increasing the volume of fluid within the film. The first simple model of injection results from assuming that the 

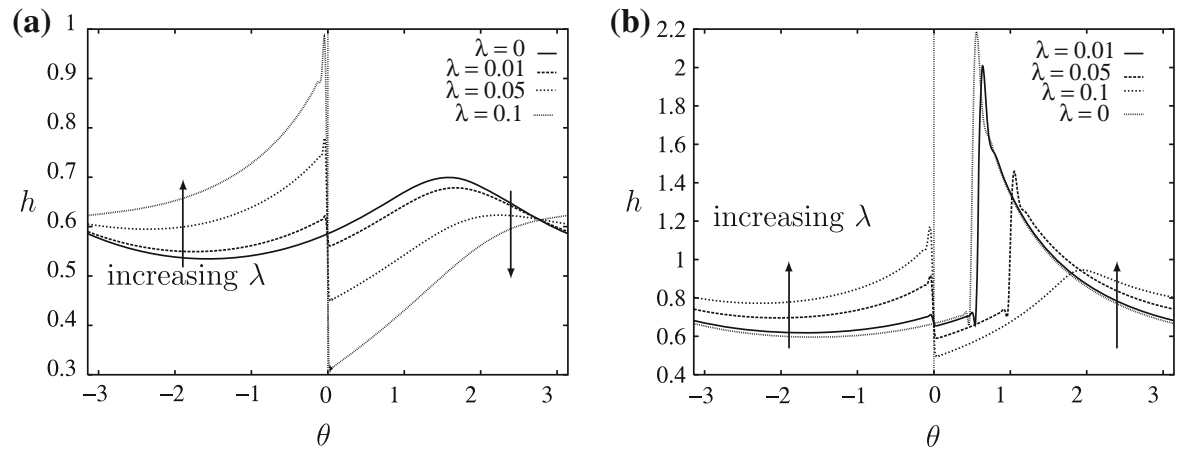

Fig. 8 Comparison of the steady-state profile of a film with uniform inflow along with a region of extraction located about $\theta=0$, for various values of $\lambda$, with $\epsilon=10^{-5}$ and $\mathbf{a} \bar{h}=0.6, \mathbf{b} \bar{h}=0.8$

distribution of impacting droplets is continuous and uniform. Thus, in this model mass is added to the flow at a fixed rate at every point on the circumference.

For a uniform inflow with an equal volume extracted through $1 \%$ of the surface at $\theta=0$ the local inflow function $I$ in (11) is given by

$I=1-100\left(H\left(\theta-\theta_{S}\right)-H\left(\theta-\theta_{e}\right)\right), \quad-\pi<\theta<\pi$,

where $H$ denotes the Heaviside function, $\theta_{s}=-\pi / 100$ denotes the start of the extraction region and $\theta_{e}=\pi / 100$ is the end of the region. Recall that $\lambda$ controls the inflow/outflow rate.

In the results that follow the film was taken to have an initial uniform thickness with a prescribed value of $\bar{h}$. The value of $\bar{h}$ then remains constant throughout the development of the film profile. A steady-state profile was assumed to have been achieved when the height of the film at each grid point at consecutive time steps was unchanged within a tolerance value of $10^{-7}$.

\subsubsection{Results}

Figure 8 illustrates steady-state profiles for moderate values of $\lambda$ with $\bar{h}=0.6$ and 0.8 . It can be seen that the height of the film falls sharply in the region of fluid extraction, with the drop in height being proportional to $\lambda$ (as expected, since the mass of fluid ahead of the sink must increase to provide the pressure head for the required balancing outflow). The location of the region of outflow prevents all the fluid from moving round to the rising side, resulting in a much thinner film there in comparison to the profiles with no inflow or outflow and a consequent thicker film on the falling side. It can be seen from Fig. $8 \mathrm{~b}$, where $\bar{h}=0.8>\bar{h}_{c 1}$, that, as a result of this, either the sharp front which forms is reduced in size, and hence positioned higher up the wall of the cylinder, or the front is prevented from forming altogether. Thus, when $\lambda$ is not small, the previous critical values of $\bar{h}$ no longer apply.

It is, however, straightforward to determine the appropriate critical values of the mean film thickness for this case. From (10) the steady-state profile with zero surface tension $(\epsilon=0)$ satisfies

$$
\frac{h^{3}}{3} \sin \theta-h+h_{0}+\lambda \int_{0}^{\theta} I(\phi) \mathrm{d} \phi=0,
$$

where $I$ is given by (15) and $h_{0}$ is the value of the height at $\theta=0$. The Implicit Function Theorem implies that a front may exist if $h^{2} \sin \theta-1=0$, or equivalently if

$$
\sin \theta=\frac{4}{9\left(h_{0}+\lambda \int_{0}^{\theta} I(\phi) \mathrm{d} \phi\right)^{2}} .
$$

For given $\lambda$, the largest value of $h_{0}$ for which no solution to (17) exists can be determined and thus the critical values of $\bar{h}$ can be obtained (through integration of the corresponding solution to (16)). For $\lambda=0.05$ the first critical value 

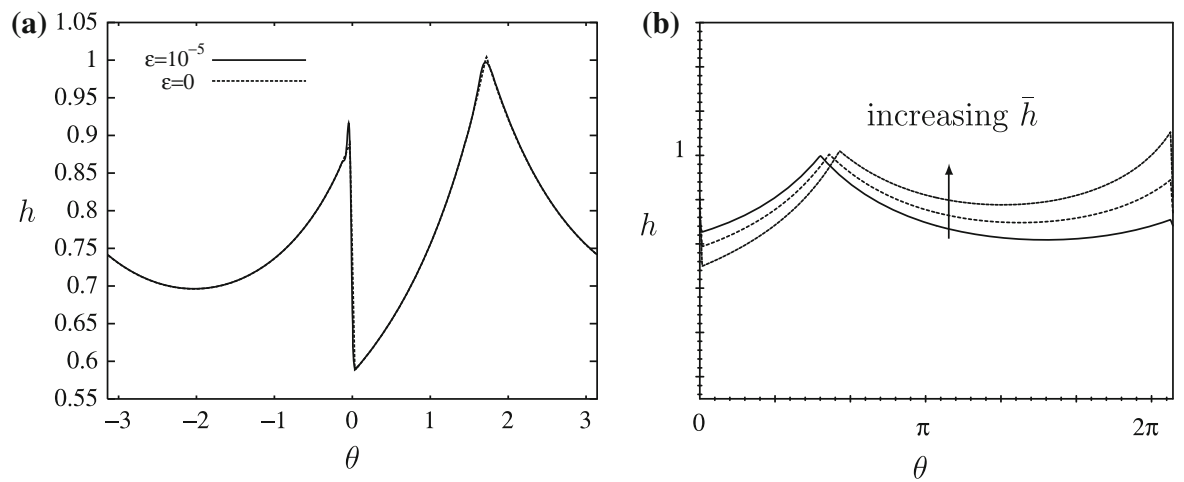

Fig. 9 Steady-state profiles of a film with uniform inflow along with a region of extraction located about $\theta=0$ : a comparison of the analytic solution for $\lambda=0.05, \epsilon=0$, with the corresponding numerical profile for $\epsilon=10^{-5}$. The average dimensionless height is $\bar{h}=\bar{h}_{c 1}=0.76484$, the critical value beyond which a sharp front will form. b The analytic solutions for the for $\lambda=0.01$ with $\bar{h}=\bar{h}_{c 1}=0.7196$ (solid line $), \lambda=0.05$ with $\bar{h}=\bar{h}_{c 1}=0.7648$ (dashed line $)$ and $\lambda=0.1$ with $\bar{h}=\bar{h}_{c 1}=0.81104($ dotted line $)$
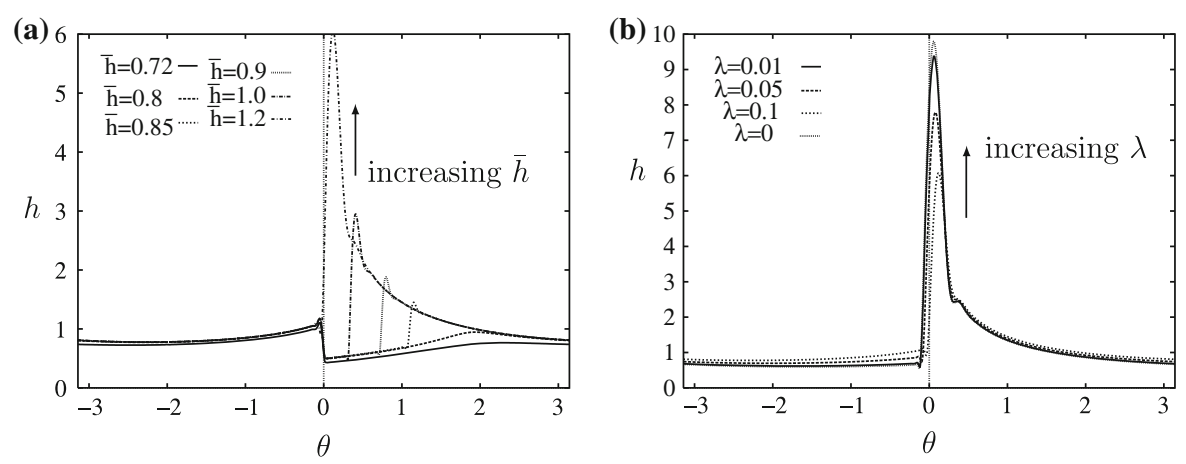

Fig. 10 Comparison of the steady-state profiles of a film with uniform inflow along with a region of extraction located about $\theta=0$, with $\epsilon=10^{-5}$, for $\mathbf{a} \lambda=0.1$ and various values of $\bar{h} ; \mathbf{b} \bar{h}=1.2$ and various values of $\lambda$

is calculated as $\hat{h}_{c 1}=0.76484$, a comparison of the numerical and analytic solutions for this case is given in Fig. 9a showing good agreement between them. It can also be seen that the position of maximum thickness occurs in the upper quadrant of the cylinder on the rising side, with $\theta_{\max }=1.7189$. Figure $9 \mathrm{~b}$ shows the analytic profiles $h(\theta)$ vs $\theta(0 \leq \theta<2 \pi)$ for three different values of $\lambda$ with $\bar{h}=\bar{h}_{c 1}$ in each case, the value of $\bar{h}_{c 1}$ increasing with the value of $\lambda$. It can be seen that the position of maximum thickness moves higher up the wall of the cylinder as $\lambda$ is increased. Thus the fluid transfer to and from the film has the effect of shifting the initial location of the front, which forms when $\bar{h}_{c 1}$ is passed, to the upper half of the cylinder, in contrast to the no-flux case where the front forms in the lower quadrant.

When $\bar{h}$ is increased beyond $\bar{h}_{c 1}$ the sharp front forms and migrates to the bottom of the cylinder as $\bar{h}$ is increased; see Fig. 10a for $\lambda=0.1$. For values of $\bar{h}$ in excess of the second critical value $\bar{h}_{c 2}$, corresponding to a front located at $\theta=0$, a pool forms at the bottom of the cylinder; see Fig. 10b. A comparison of these profiles with those experiencing no inflow or outflow for the same value of $\bar{h}$ shows the uniform inflow to affect the distribution of fluid around the cylinder. Figure 10b shows the film for $\lambda \neq 0$ has a thicker film around the cylinder's surface and a shallower pool than that for $\lambda=0$. It should be noted that the second critical values of $\bar{h}$ corresponding to this figure are $\bar{h}_{c 2}=1.121$ for $\lambda=0.01, \bar{h}_{c 2}=1.1923$ for $\lambda=0.05$ and $\bar{h}_{c 2}=1.274$ for $\lambda=0.1$; thus the front in the latter profile has not yet reached the bottom of the cylinder.

The effects of increasing surface tension (not illustrated) are the now-familiar smoothing of the flat front and the pool; in addition, the 'discontinuity' at the outflow point (if present) is also smoothed. 
Fig. 11 The input function $I_{I}$ used to approximate the findings of [19]

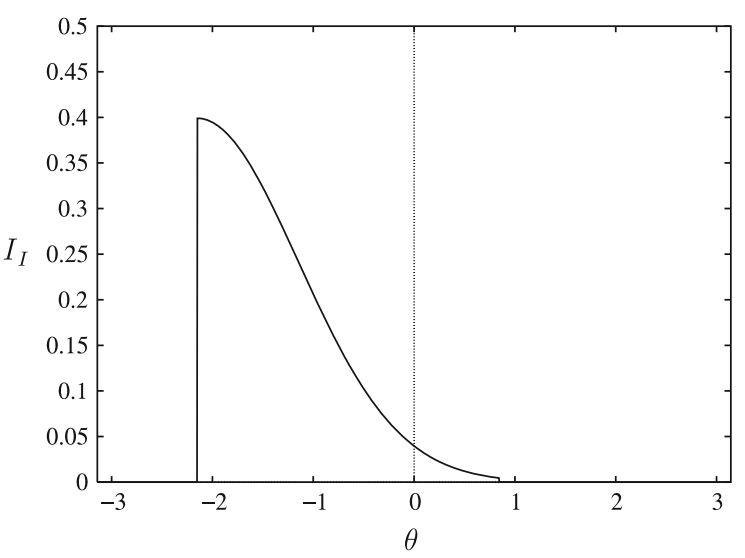

5.2 Non-uniform injection with a fixed point of extraction

In practice it is unlikely that the distribution of droplets impacting on the film is uniform as assumed above. Droplets of various sizes will be present and consequently they will follow different paths around the chamber with the motion of smaller drops being determined largely by the air flow, while that of the larger drops motion is strongly influenced by gravity.

Farrall [19], in a numerical study of bearing-chamber fluid flows, tracked the trajectory of droplets released into an annular rotating air flow, the air flow being induced by a central rotating cylinder, the outer cylinder being stationary. The droplets were released in the top region of the annulus (corresponding to $\theta=\pi$ ) with a prescribed initial horizontal velocity. The droplets were then tracked until they impacted on the outer stationary cylindrical wall. In the absence of experimental evidence, a Rossin-Rammler distribution for the size distribution of droplets was assumed; see [27]. For a range of distribution parameters and injection velocities, the results showed that the droplets which reached the outer wall did so in the region $-2.14 \leq \theta \leq 0.86$, relative to the present coordinate system. The larger the droplets, the higher up the cylinder wall they impacted and thus a large proportion of the mass inflow is in the region of $\theta=-2.14$, with only a small amount distributed over the remainder of the region.

These results may be incorporated into the film-flow model to give a more representative description of the droplet flux. Obviously, for the case of rimming flows on a rotating surface, the motion of the outer wall would have the effect of increasing the rotating airflow within the annulus. It is likely that this would increase the spread of the droplets around the cylinder, smoothing out the mass-distribution results obtained by Farrall [19]. To reflect this, in the third injection model, the input function $I_{I}$ is taken to be given by

$I_{I}(\theta, t)=\frac{1}{\sigma \sqrt{2 \pi}} \mathrm{e}^{-\frac{(\theta+2.14)^{2}}{2 \sigma^{2}}}[H(\theta+2.14)-H(\theta-0.86)], \quad-\pi \leq \theta \leq \pi$.

This distribution is illustrated in Fig. 11 and has the same qualitative form as the volume distributions at the cylinder wall identified by Farrall [19]; in all the following analysis $\sigma=1$ to ensure that the semi-normal curve fits in the specified injection region of width 3 radians. Thus, the area beneath the curve (18) is 0.4985 . As in the previous section, a thin uniform film of thickness $\bar{h}=10^{-7}$ is introduced to prevent numerical instabilities developing in the initial stages of the film evolution.

The inflow function $I_{I}$ may be extended by removing the added mass through an outflow region about $\theta=0$ as in the previous section. This results in the following representation for the function $I$,

$I(\theta)=\frac{1}{\sqrt{2 \pi}} \mathrm{e}^{-\frac{1}{2}(\theta+2.14)^{2}}[H(\theta+2.14)-H(\theta-0.86)]-A\left[H\left(\theta-\theta_{o s}\right)-H\left(\theta-\theta_{o e}\right)\right], \quad-\pi \leq \theta \leq \pi$,

where $\theta_{o s}=-\pi / 100$ and $\theta_{o e}=\pi / 100$ mark the start and end of the outflow region and $A \approx 7.934$ is the rate at which fluid must be extracted to maintain a constant volume. To ensure conservation of mass in the numerical results, the outflow rate is calculated at the first iteration within the program by summing the inflow at each grid 

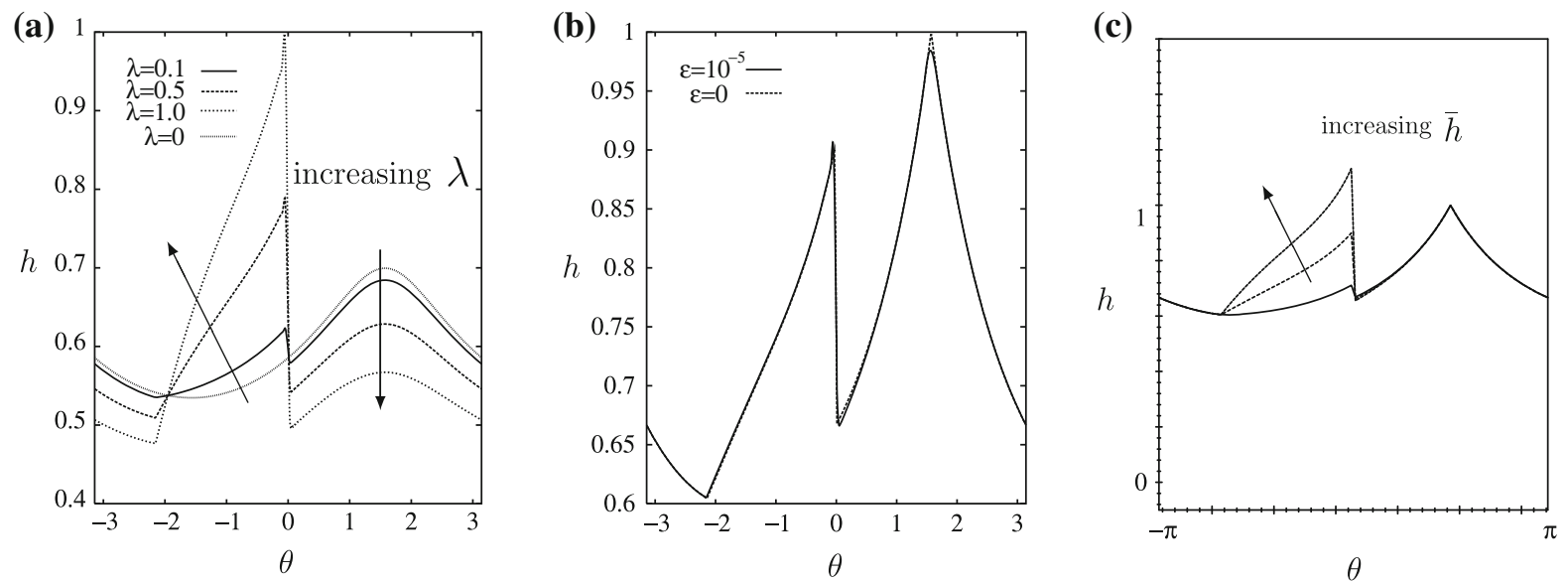

Fig. 12 Profiles of films with non-uniform inflow over a region and with an outflow section located at $\theta=0$ : a numerical results for various values of $\lambda$, with $\bar{h}=0.6$ and $\epsilon=10^{-5} ; \mathbf{b}$ comparison of the analytic solution $(\epsilon=0)$ with the corresponding numerical profile for $\epsilon=10^{-5}, \bar{h}=\bar{h}_{c 1}=0.74904$ and $\lambda=0.5$; $\mathbf{c}$ analytic solutions for $\lambda=0.1$ with $\bar{h}=\bar{h}_{c 1}=0.71575$ (solid line), $\lambda=0.5$ with $\bar{h}=\bar{h}_{c 1}=0.74904$ (dashed line) and $\lambda=1.0$ with $\bar{h}=\bar{h}_{c 1}=0.78770$ (dotted line)

point and dividing by the total number of grid points in the extraction region. Steady-state profiles are then sought using the criteria for convergence given in Sect. 5.1.

\subsubsection{Results}

It can be seen from Fig. 12a, where $\bar{h}=0.6$, that, due to the position of the injection and extraction regions, the profile has the appearance of one of the steady-state flows with no inflow/outflow, except for an additional volume of fluid located on the falling side of the cylinder in the region of inflow. This extra volume of fluid along with the height of the front at the point of outflow is seen to increase with the value of $\lambda$, as expected.

For larger values of $\bar{h}$ the front forms on the lower rising side, or if $\bar{h}$ is sufficiently large, a pool develops at the bottom of the cylinder. As in Sect. 5.1, the critical values of $\bar{h}$ which relate to these profile formations may be determined through analysis of the governing profile equation (10) with $\epsilon=0$. The results show good agreement with the numerics as can be seen in Fig. $12 \mathrm{~b}$ for $\lambda=0.5$ with $\bar{h}_{c 1}=0.74904$. Figure 12c shows analytic profiles for different values of $\lambda$ with $\bar{h}=\bar{h}_{c 1}$ in each case. It can be seen that $\bar{h}_{c 1}$ increases with $\lambda$, though, unlike the inflow regime discussed in Sect. 5.1, the transfer of fluid has not affected the position of maximum film thickness with it being located at $\theta=\pi / 2$ in all cases.

When $\bar{h}$ is increased beyond $\bar{h}_{c 1}$ the front forms in the usual manner, with its position being lower in the cylinder for larger values of $\bar{h}$, relative to $\bar{h}_{c 1}$; see Fig. 13a. When the second critical value of $\bar{h}$ (which also depends on $\lambda$ ) is exceeded, the pool forms in the usual manner at the bottom of the cylinder, as may be inferred from Fig. 13b. The film is seen to thicken on the falling side of the cylinder in the region of the inflow as $\bar{h}$ increases.

By increasing the Bond number $\epsilon$ the discontinuity at the point of extraction may be smoothed out, along with the flat front which forms on the lower rising side (not illustrated).

\section{Conclusions}

In order to develop an understanding of some of the phenomena that may feature within a bearing chamber, a simple mathematical model incorporating various regimes of fluid injection and extraction has been applied to rimming films. Although it is possible to construct many different regimes of fluid transfer to and from the films, this initial scoping study has been restricted to those which have some relevance to flows within a bearing chamber. The results 

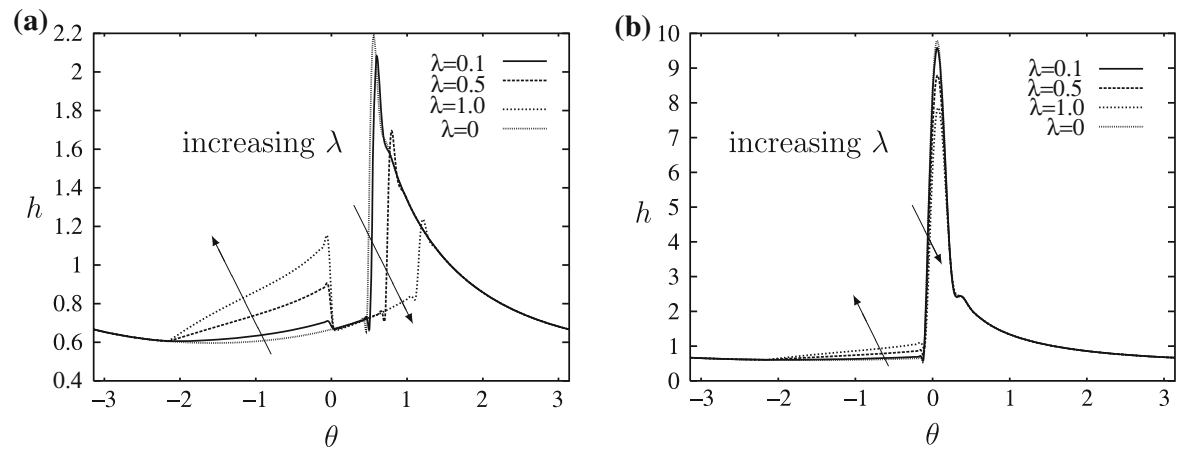

Fig. 13 Comparison of the steady-state profiles of a film with uniform inflow along with a region of extraction located about $\theta=0$, with $\epsilon=10^{-5}$, for various $\lambda$ : $\mathbf{a} \bar{h}=0.8\left[\bar{h}_{c 1}=0.71575\right.$ for $\lambda=0.1, \bar{h}_{c 1}=0.74904$ for $\lambda=0.5$ and $\bar{h}_{c 1}=0.78770$ for $\left.\lambda=1.0\right]$; $\mathbf{b} \bar{h}=1.2\left[\bar{h}_{c 2}=1.111\right.$ for $\lambda=0.1, \hat{h}_{c 2}=1.145$ for $\lambda=0.5$, and $\bar{h}_{c 2}=1.184$ for $\left.\lambda=1.0\right]$

show that the profiles in all of the scenarios studied have the same qualitative form as those seen in Sect. 3 for classical rimming flow without injection or extraction, although the detailed profile observed is dependent on the particular regime of inflow/outflow being studied.

If the volume of fluid within the film is held constant through the extraction of the fluid which is added, the film profile achieves a steady-state. The film profile along with critical values of the average dimensionless film thickness $\bar{h}$, for which a steep front $\left(\bar{h}_{c 1}\right)$ or pool $\left(\bar{h}_{c 2}\right)$ begins to form, may be determined through analysis of the governing profile equation when surface tension is neglected $(\epsilon=0)$. In both of the inflow regimes studied, $\bar{h}_{c 1}$ and $\bar{h}_{c 2}$ were found to increase with $\lambda$ so that more fluid may be held in a film without the formation of a front for larger values of $\lambda$, thus reducing the chances of oil being stripped away by the core flow in a bearing chamber. Additionally, in the case of a uniform inflow with a point extraction, the initial location of the steep front was displaced to a position higher in the cylinder.

Associated with the formation of a steep front is the development of a recirculating pool, which, as stated in Sect. 4, is undesirable in an aero-engine, since the recirculation inhibits heat transfer. The model, however, provides a means of investigating whether the formation of such pools can be delayed, or inhibited altogether, by designing jets to deliver prescribed distributions of oil droplets or by the judicious siting of oil sinks. Moreover, by studying pathlines, oil-residence times can be predicted and systems optimized to enhance heat transfer. The focus of the current study is, however, purely on mass-transfer effects and potentially important momentum effects are totally neglected. Thus, the usefulness of the present model as a design tool for aero-engines is restricted and the next step is to develop a combined mass and momentum transfer model.

Acknowledgements CJN gratefully acknowledges the financial support of the University of Nottingham and the EC (BRPR-CT970539 INTRANS). The authors would to thank the referees for their constructive comments.

Open Access This article is distributed under the terms of the Creative Commons Attribution Noncommercial License which permits any noncommercial use, distribution, and reproduction in any medium, provided the original author(s) and source are credited.

\section{References}

1. Thoroddsen ST, Mahadevan L (1997) Experimental study of coating flows in a partially filled horizontal rotating cylinder. Exp Fluids 23:1-13

2. Moffatt HK (1977) Behaviour of a viscous film on the outer surface of a rotating cylinder. J Mec 16:651-673

3. Pukhnachev VV (1977) Motion of a liquid film on a surface of a rotating cylinder in a gravitational field. J Appl Mech Tech Phys 18:344-351

4. Johnson RE (1988) Steady-state coating flows in a rotating horizontal cylinder. J Fluid Mech 190:321-342

5. O'Brien SBG, Gath EG (1998) The location of a shock in rimming flow. Phys Fluids 10:1040-1042 
6. Ashmore JJ, Hosoi AE, Stone HA (2003) The effect of surface tension on rimming flows in a partially filled rotating cylinder. J Fluid Mech 479:65-98

7. Benjamin TB, Pritchard WG, Tavener SJ (1993) Steady and unsteady flows of a highly viscous liquid inside a rotating horizontal cylinder. Preprint

8. Hosoi AE, Mahadevan L (1999) Axial instability of a free-surface front in a partially filled horizontal rotating cylinder. Phys Fluids 11:97-106

9. Noakes CJ (2001) The dynamics of liquid films on rotating surfaces. PhD Thesis, University of Nottingham

10. Tirumkudulu M, Acrivos A (2001) Coating flows within a rotating horizontal cylinder: lubrication analysis, numerical computations, and experimental measurements. Phys Fluids 13:14-19

11. Wilson SK, Hunt R, Duffy BR (2002) On the critical solutions in coating and rimming flows on a uniformly rotating horizontal cylinder. Q J Mech Appl Math 55:357-387

12. Benilov ES, O’Brien SBG (2005) Inertial instability of a liquid film inside a rotating horizontal cylinder. Phys Fluids 17:1-16

13. Noakes CJ, King JR, Riley DS (2006) On the development of rational approximations incorporating inertial effects in coating and rimming flows. Q J Mech Appl Math 59:163-190

14. Kelmanson MA (2009) On inertial effects in the Moffatt-Pukhnachov coating-flow problem. J Fluid Mech 633:327-353

15. Hinch EJ, Kelmanson MA (2002) On the decay and drift of free-surface perturbations in viscous thin-film flow exterior to a rotating cylinder. Proc R Soc A 459:1193-1213

16. Noakes CJ, King JR, Riley DS (2005) On three-dimensional stability of a uniform rigidly rotating film on a rotating cylinder. Q J Mech Appl Math 58:229-256

17. Groh CM, Kelmanson MA (2009) Multiple-timescale asymptotic analysis of transient coating flows. Phys Fluids 21:091702

18. Villegas-Díaz M, Power H, Riley DS (2005) Analytical and numerical studies of the stability of thin-film rimming flow subject to surface shear. J Fluid Mech 541:317-344

19. Farrall M (2001) Numerical modelling of two-phase flows in a simplified bearing chamber. PhD Thesis, University of Nottingham

20. Farrall M, Hibberd S, Simmons K, Giddings D (2006a) Prediction of air/oil exit flows in a commercial aero-engine bearing chamber. J Aerodyn Eng 220:197-202

21. Farrall M, Hibberd S, Simmons K, Gorse P (2006b) A numerical model for oil film flow in an aeroengine bearing chamber and comparison to experimental data. J Eng Gas Turbines Power-Trans ASME 128:111-117

22. Benilov ES, Benilov MS, Kopteva N (2008) Steady rimming flows with surface tension. J Fluid Mech 597:91-118

23. Schiesser WE (1991) The numerical method of lines. Academic Press, San Diego

24. Black GJB (2002) Theoretical studies of thin-film flows. MPhil thesis, University of Strathclyde

25. Benilov ES, Benilov MS, O'Brien SBG (2009) Existence and stability of regularized shocks, with applications to rimming flows. J Eng Math 63:197-212

26. Wilson SDR, Williams J (1997) The flow of liquid on the inside of rotating cylinder and some related problems. Phys Fluids 9:2184-2190

27. Mugele RA, Evans HD (1951) Droplet size distribution in sprays. Ind Eng Chem 43:1317-1324 